\title{
LA-UR $92-1529$
}

LA-UR--92-1529

DE92 015276

\section{TITLE Problems Associated with Launching Hypervelocity Projectiles from the Fast Shock Tube}

AUTHOR(S) Jerry F. Kerrisk and John K. Meier

SUBMITTED to The 1992 Hypervelocity Impact Symposium

November 17-20, 1992

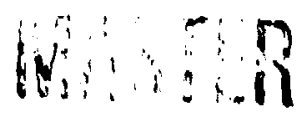

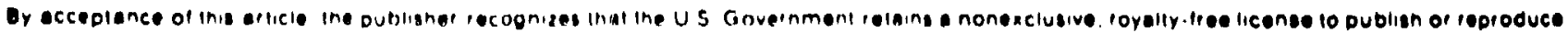

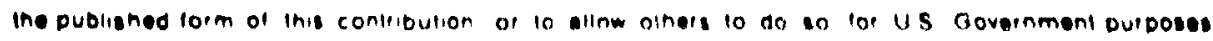

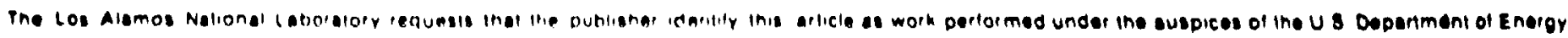

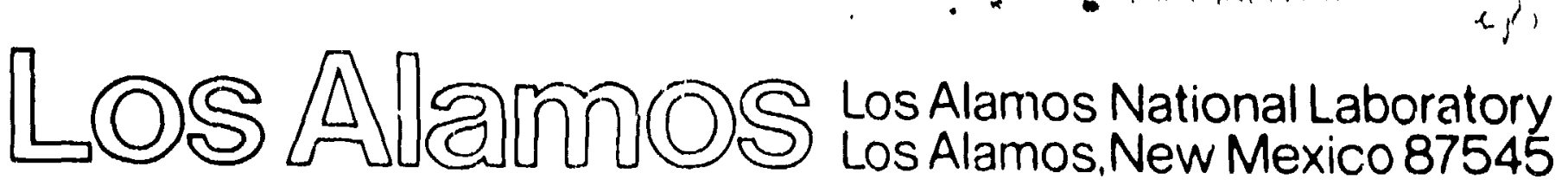




\title{
Problems Associated with Launching Hypervelocity Projectiles from the Fast Shock Tube
}

\author{
Jerry F. Kerrisk and John K. Meier \\ Los Alamos National Laboratory \\ Mail Stop F663 \\ Los Alamos New Mexico 87545
}

\begin{abstract}
Modeling and experiments are being done with the goal of understanding the physics of projectile acceleration at high driving pressures (megabar range) and short acceleration times (a few microseconds) well enough to design and test successiul hypervelocity launch systems. The Fast Shock Tube, a cylindrically convergent high-explosive driver, has been used to accelerate projectiles.

Detailed modeling of the experiments, including high-pressure gas flow, projectile instability, and projectile fracture, has been done with MESA/2D. Modeling results show quantitative agreement with the average behavior of the system. However, detaiis of projectile behavior are not predicted well. Observed velocity distributions across the diameter of a projectile or projectile shapes are only in qualitative agreement with calculations. This, then, presents the major constraint on the successful design of a launch system: that the processes that limit projectile integrity depend on the details of the drive conditions, and these details are not quantitatively modeled at this time.
\end{abstract}

\section{INTRODUCTION}

The Fast Shock Tube (FST) is a cylindrically convergent high-explosive (HE) system for driving a flat axial shock in a polystyrene foam core inside the HE 
(Marsh and Tan, 1991; Meier and Kerrisk, 1991; Kerrisk and Meier, 1991) The shocked foam, which acts like a gas, is used as a driver for gas-flow or projectileacceleration tests. Drive conditions much higher than direct HE drive can be obtained in the FST. Projectiles can be shock accelerated (in direct contact with the foam) or accelerated more gently by expanding the foam before acceleration. Peak driving pressures on flat-plate projectiles have ranged from 0.3-1 Mbar. Intact plates 'iave been accelerated up to $\sim 0.9 \mathrm{~cm} / \mu \mathrm{s}$ over distances of a few centimeters.

The work discussed here represents an attempt to probe limits of projectile acceleration under the high-pressure drive conditions attainable in the FST. The initial problem, that of achieving a source of high-pressure gas with unifcrm conditions (pressure, density, velocity) over an area of at least a few square centimeters, has been solved by the FST driver (Meier and Kerrisk, 1991; Menikoff et al., 1991). However, the use of this driver gas presents a number of additional problums. If projectile acceleration were one dimensional, problems of shuck formation and spall fracture would still occur. These problems can be mitigated by tailoring the pressure/time history of the drive with an expansion region between the initial location of the drive gas and the projectile. However, one-dimensional drive is an oversimplification. With a uniform, two-dimensional drive but with ideal (rigid, reflecting) boundaries, the additional problems of projectile instability, stresses associated with exit from a barrel, and twodimensional fracture would occur. These problems become more difficult to solve as the driving pressure increases. With the FST, however, even this is an ovorsimplification. Driving pressures in the FST are well above any material strengths. Thus ieal boundaries (for example, the barrel wall) deform during projectile acceleration. Deformation of boundaries leads to flow perturbations 
and nonuniform drive conditions. This, in turn, intensifies the instability and fracture problems inherent in the two-dimensional system.

These problems are being investigated through a combination of experiments and two-dimensional modeling. Our goal is to understand the physics underlying the process of projectile acceleration with a high-pressure gas well enough to design and test successful launch systems.

\section{DESCRIPTION OF THE FAST SHOCK TUBE}

Figure 1 shows one of a number of related FST systems that has been built and tested. The initiator section insures a uniform initiation of the driver HE around the outer periphery. The detonation front moves axially along the HE/foam interface in the driver at the detonation velocity $(0.88 \mathrm{~cm} / \mu \mathrm{s}$ for PBX 9501$)$, forming a flat, normal shock in the foam. The design parameters of the driver section (diameters, lengths, type of $H E$, foam density) must be chosen properly to achieve the flat shock and uniform flow conditions behind the shock in the foam core (Meier and Kerrisk, 1991; Menikott et al., 1991). In the system of Fig. 1 , an initial foam density of $0.5 \mathrm{~g} / \mathrm{cm}^{3}$ gives a pressure of $-0.3 \mathrm{Mbar}$ and a particle velocity of $\sim 0.66 \mathrm{~cm} / \mu \mathrm{s}$ behirid the shock in the foam. Figure 2 shows calculated material interfaces and pressure contours in the HE and foam of the driver from Fig. 1 when the shock is $\sim \mathrm{cm}$ from the end of the driver. The calculations (all hydrodynamic calculations discussed here were done with MESA/2D (Cagliostro et al., 1990)) are in good agreement with radiographs showing the detonation front and flat shock in the foam. The lack of radial variation of the pressure (also density and axial velocitv) contours behind the shock shows that this flow is radially uniform. This condition is necessary to achieve a sustained, uniform acceleration. 
An experimental section consisting of a barrel, an expansion region for the shocked foam, and a projectile (plate) is also shown in Fig. 1. A variety of other experiments including driver diagnostics or direct acceleration of plates have bfen used on the end of the driver. Projectiles in the form of plates of stainless steel, Ti, and Ta with diameters of $10-25 \mathrm{~mm}$ and thicknesses of $0.7-3.5-\mathrm{mm}$ have been accelerated.

\section{EXPANSION OF THE DRIVING GAS}

Projectiles can be accelerated by placing the projectile in direct contact with the foam. However, this method shocks the projectile up to pressures of $\sim 1$ Mbar. Although these shock pressures are not high enough to melt the maerials tested, they result in high temperatures and the possibility of spalling. For these reasons, a short expansion down a barrel has been used to modity the pressure/time history driving the plate (Fig. 1). Although expansion can eliminate shocks in the projectile, the high pressures and velocities of the expanding foam deform the barrel walls during expansion and acceleration. Flow past the deformed walls disturbs the initially uniform flow from the driver and results in increasingly nonuniform drive conditions. Figure 3 shows material interfaces and vector velocities in the vicinity of the barrel (stainless steel) and projectile (2- $\mathrm{mm}$ thick stainless steel) $\sim 5 \mu$ after the start of motion of the projectile. The distorted barrel walls (compare with Figs. 1 and 2) and radially nonunitorm flow behind the projectile are evident.

MESA/2D calculations of initially uniform flow entering and flowing down a stainless steel barrel show flow perturbations starting at the barrel wall and moving radially out into the gas stream. The most prominent deformations of the barrel wall occur at the entrance, at any discontinuities such as the interface between the foam and expansion region, and at the initial axial location of the 
plate (see Fig. 3). The design of the barrel entrance shown in Fig. 1 represents an attempt to minimize flow perturbations from the entrance.

Flow that is essentially uniform $1-2 \mu \mathrm{s}$ after the start of expansion develops density perturbations of $10-20 \%$ by $5 \mu$ s after the start of flow. The magnitude of a perturbation also tends to increase as it converges radially. These perturbations result in nonuniform drive conditions. The details of when and where (radially) the flow perturbations influence the plate depend, among other parameters, on the sound speed in the shocked and expanding foam. Attempts to calculate the effects of these flow perturbations on projectile velocity have shown only qualitative agreement with observations ( $s e \theta$ discussion of LINE VISAR measurements, below). Uncertainties in the equation of state (EOS) of the foam are thought to be mostly responsible for these differences.

\section{EARLY PROJECTILE ACCELERATION}

The early motion of 2-mm thick stainless steel plates has been observed in systems like Fig. 1 using a LINE VISAR to measure velocity across a diameter of the plate (Hemsing et al., 1991). This technique provides velocity data for $2.4 \mu \mathrm{s}$ after the start of motion. After that time, gas blowby around the edges of the plate disrupts the VISAR signal. Figure 4 shows plots of the axial velocity of the front surface of the plate as a function of the distarice across a diameter. At each time an obşrved and calculated velocity profile are shown. At $50.5 \mu \mathrm{s}(\sim 0.1 \mu \mathrm{s}$ after the start of motion), the observed data show the edges of the plate moving faster than the center; the calculations show the center moving faster. By $50.9 \mu \mathrm{s}$, the observed and calculated velocities are in good agreement. At later times, some show good agreement between observations and calculations and others show poor agreement. 
An examination of the observed velocity profiles in Fig. 4 shows that a perturbation (an increase in velocity) starts at the edges of the plate at $\sim 50.7 \mu \mathrm{s}$ and moves toward the center, reaching the $r=0$ axis between 51.6 and $52 \mu s$. This disturbance in the velocity is probably caused by a flow perturbation in the driving gas. The calculations indicate a much smaller perturbation of a similar nature. The discrepancy between these observations and calculations is an examp!e our inability to calculate the details of the flow perturbations and their effects on the plate at this time.

Calculated average projectile velocities depend to a large extent on the EOS of the foam. Figure 5 is a plot of calculated projectile veiocity $(2-\mathrm{mm}$ thick by 25-mm diameter stainless steel plate) using ideal-gas and SESAME (Holian, 1984) EOSs for the foam cornpared with the measured average velocities obtained from three radiographs and impact on a witness plate. These two EOSs were chosen based on comparisons of the observed and calculated shock positions in the FST driver. In that comparison and for projectile velocities, they tend to bracket the observed behavior. A single suitable EOS has not been found.

\section{PROJECTILE STABILITY}

During acceleration, the driving gas that is pushing the projectile is of lower density than the projectile material. This leads to the possibility of instabilities that are similar to the Rayleigh-Taylor instability at the interface between two fluids of different density in a gravitational field. Previous examinations of the stability of accelerated plates have done Lagrangian numerical calculations (Swegle and Robinson, 1989) or experiments (Barnes et al., 1974; Barnes et al., 1980 ) on plates with prescribed initial surface perturba:ions. This work would be applicable if the driving pressure were uniform across the diameter of a plaie. 
However, experiments and calculations indicate that nonuniformities exist in the FST drive. The calculations described here were Eulerian (with MESA/2D, the same code used for other FST calculations) and used a prescribed drivingpressure perturbation on a plate that had an initialiy uniform surface.

Perturbations that only varied spatially and that varied with space and time were used.

A group of calculations was done initially to compare the MESAV2D results with the Lagrangian calculations of Swegle and Robinson (1989). The agreement was good except in one case where the system was on the boundary between stability and instability. Swegle and Robinson predicted the plate was just stable, but MESA2D predicted it was marginally unstable. Trends in the susceptibility of plates to instability were observed from calculations in which various parameters were systematically varied. Increasing the wavelength to plate thickness ratio, increasing the relative mas itude of the perturbation, increasing the driving pressure, or decreasing the yield strength of the plate all led to greater tendency for instability. These same trends were ot - วrved whether produced by an initial surface perturbation on the plate or by a driving pressure perturbation.

A simple analytical argument can be made to show that these two types of perturbations are equivalent after an initiation period. In the limit of small perturbations and for a steady-state pressure gradient, there is a relation between the initial relative surface perturbation $\left(\Delta h_{0} / h\right)$ and the relative pressure perturbation ( $\Lambda P / P)$ that give the same axial pressure gradient. For this analysis, $h$ is the plate thickness, $\Delta h$ is the peak-to-peak surface variation, $\Delta h_{0}$ is the initial value of $\Delta h, P$ is the pressure, and $\Delta P$ is the peak-to-peak pressure variation. Figure 6 .hows sketches of the pressure gradient through a plate of thickness $h$. On the left, the driving pressuis is uniform and the gradients for two different 
plate thicknesses $\left[h\right.$ and $\left(h-\Delta h_{0}\right)$ ] are shown. On the right, the plate thickness is uniform and the gradients for two different driving pressures $[(P+1 / 2 \Delta P)$ and $(P-1 / 2 \Delta P)]$ are shown. For the ratio of these two gradients to be the same in the two cases, Fig. 6 shows that

$$
\Delta h_{0} / h=\Delta P / P \text {. }
$$

That is, the ratio of the pressure gradients would be the same if the magnitude of the relative initial surface perturbation $\left(\Delta h_{0} / h\right)$ is the same as the magnitude of the relative pressure perturbation $(\Delta P / P)$. Because the axial pressure or stress gradient drives the instability (Swegle and Robinson, 1989), the behavior of plates should be similar under these conditions.

Figure 7 is a plot of $\Delta \mathrm{n}$ as a function of time that compares three calculations in which a uniform drive pressure and initial surface perturbation were used $\left(\Delta h_{0} / h=0.2 \%\right.$ ) with three otherwise identical calculations in which a uniform plate and a perturbation $(\triangle P / P=0.2 \%$ ) in the driving pressure were used. For these calculations, the average driving pressure for a $2-\mathrm{mm}$ thick $W$ plate rose to 1 Mbar in 0.1, 1.0, or 10.0 us and then was held constant. At early time there is a large difference between the two sets of calculations because it takes some time for plates that have $\Delta h_{0}=0$ to catch up with the plates the have $\Delta h_{0}=0.0004 \mathrm{~cm}$. At later time, the best agreement is for the problem with the longest rise time $(10 \mu \mathrm{s})$; this problem hest meets the assumption of a steadystate pressure gradient. However, the agreement is reasonable in the case of the shortest rise time $(0.1 \mu \mathrm{s})$ even though the pressure gradient is far from steady state. The problem with the intermediate rise time $(1 \mu \mathrm{s})$ is on the boundary between stability and instability and shows the poorest late-time agreement of the three. 
Stability calculations that are more characteristic of the type of perturbations seen in the FST were also done. For these calculations the average driving pressure on a $24-\mathrm{mm}$ diameter by $2-\mathrm{mm}$ thick stainless steel plate rose to a maximum $\left(P_{m}\right)$ oi $0.4,0.7$, or $1 \mathrm{Mbar}$ in $1 \mu \mathrm{s}$, held constant for $1 \mu \mathrm{s}$, and then dropped to zero over $6 \mu \mathrm{s}$. A pressure perturbation that started near the edge of the plate and moved toward the $r=0$ axis at $0.5 \mathrm{~cm} / \mu$ s was superimposed on the average pressure. The perturbation was assumed to decay to zero after it reached the $r=0$ axis. A series of calculations was done to find the magnitude of the pressure perturbation $(\Delta P / P)$ that produced the same maximum plate deformation $\left(\Delta h_{m}\right)$ at a particular time in the calculation. Figure 8 is a plot of $\Delta P / P$ as a function of $P_{m}$ that produces $\Delta h_{m}=0.5 \mathrm{~mm}$ at $12 \mu \mathrm{s}$ and $\Delta h_{m}=5 \mathrm{~mm}$ at $12 \mu \mathrm{s}$. The lines represent contours of constant $\Delta h_{m}$ in $\Delta P / P-P_{m}$ space. The velocities shown are the final plate velocities. Figure 8 indicates that as the maximum driving pressure in the FST increases, the allowable $\triangle P / P$ for some fixed maximum plate deformation decreases. The relation is nonlinear so that the decrease in allowable $\triangle P / P$ is occurring faster than the proportionate increase in pressure. Larger levels of $\triangle P / P$ can be tolerated if larger maximum plate deformations are acceptable.

\section{PROJECTILE INTEGRITY}

Radiographs have often shown breakup of projectiles that have exited the barrel of the FST (Marsh and Tan, 1991). This breakup is probably caused by conditions (time-varying, localized tensile stresses) within the projectile that lead to fracture. The ability of two fracture models in MESA/2D to describe this behavior was examined with calculations of the acceleration of a $19-\mathrm{mm}$ diameter by $2.66-\mathrm{mm}$ thick Ti-6Al-4V plate. This material was highiy fragmented in test radiographs (Marsh and Tan, 1991). Parameters for the Johnson-Cook Damage 
(JCD) model (Johnson and Cook, 1985) were originally obtained from Johnson and Holmquist (1989). However, use of these parameters to model a onedimensional spall test (Me-Bar et al. 1987) did not predict spall when it was observed. Values of $\sigma_{\text {spall }}$ and $\theta_{\min }$ were varied in a series of one-dimensional calculations until the calculated stress matched the observed data. The other model parameters $\left(D_{1}-D_{5}\right)$ were held fixed at the values given by Johnson and Holmquist. Parameters for the Johnson Spall (JS) model (Johnson, 1981) were determined by matching the same spall-test data.

As a measure of damage, the JCD model uses a damage fraction $(0 \leq D \leq 1)$ that is accumulated from the ratio of the incremental plastic strain to a predicted strain to failure at each time step. The JS model calculat.9s a material porosity $(0 \leq \alpha \leq 1)$. Although the ranges of these two damage measures are the same, they are probably not directly comparable. There is also a difference in how damage is communicated to the strength model. With the JCD model, there is no communication until $D=1$, at which time the yield strength and shear modulus are set to zero. In the JS model, the yield strength and shear modulus are continuously degraded as porosity increases (Johnson, 1981).

Figure 9 shows a plot of plastic strain, damage fraction, and porosity at $r=0$ and the mid plane of the plate for three calculations of the acceleration of the Ti-6Al-4V prate, one without fracture, one using the JS model, and one using the JCD model. The accumulated plastic strain difiers signiticantly among the three calculations. With the ICD model, plastic strain is no longer accumulated after $-23.1 \mu \mathrm{s}$, wnen $D$ is 1 at this location. (The earlior rise of $D$ above 1 (at 20.5 $\mu \mathrm{s}$ ) and its subsequent drift downward were caused by numerical diffusion in this Eulerian calculation.) Although there is a difference in the magnitude of damage fraction and porosity, the two models tend to show sharp increases at about the 
same time. This is when the local tensile stresses are highest. Looking at damage patterns over the entire plate, the two models predict the most damage in the same regions, near the axis $(r=0)$ toward the front of the plate (where spall is mos: likely) and near the intersection of the plate with the barrel walls (where considerable plastic strain occurs). Neither model predicts the complete fragmentation observed in the test.

\section{LIMITATIONS ON PROJECTILE ACCELERATION}

The roal of this work is to understand the physics underlying projectile acceleration well enough to design and test successful hypervelocity launch systems. A qualitative understanding of the problems associated with projectile acceleration at high driving piessures and short acceleration times has been attained. The major problems encountered have been shock formation in the projectile, projectile instability, and projectile fracture. Shock formation in the projectile can be limited by tailoring the pressure/time profile on the projectile. In the FST, an expansion region between the foam core and the projectile limits shock formation. However, flow in the exnansion region leads to flow perturbations that result in nonuniform driving pressures on the projectile. The likelihood of instabilities leading to projectile distortiun or breakup increases as the drive becomes more nonuniform. Projectile fragmentation, probably from localized tensile stresses, has been shown to be a material-dependent problem (Marsh and Tan, 1991).

Modeling of FST tests has shown quantitative agreement with the average behavior of the system. For example, calculated average projectile velocities are normally in good agreement with observations. However, details of projectile behavior are not predicted well. Observed velocity distributions across the diameter of a projectile (LINE VISA.R data) or projectile shapes (radiographs) are 
only in qualitative agreement with calculations. This presents the major constraint on the successful design of a launch system: that the processes that limit projectile integrity depend on the details of the drive conditions, and these details are not quantitatively modeled at this time. The need for two-dimensional modeling is a consequence of the kinds of problems encountered. The nonuniformities in driving pressure, material distortions, and plate instabilities and fracture are all two-dimensional effects that would be missed in one-dimensional modeling.

\section{ACKNOWLEDGMENTS}

The authors would like to thank other researchers at Los Alamos National Laboratory for their assistance in this viork. They include M. George, W. Hemsing, M. Holder, and S. Marsh for their test data; and J. Shaner, T. H. Tan, and R. Godwin for their comments and support.

\section{REFERENCES}

Barnes, J. F., P. J. Blewett, R. G. McQueen, K. A. Meyer, and D. Venable (1974). Taylor instability in solids. J. /.ppl. Phys., 45, 727-732.

Barnes, J. F., D. J. Janney, R. K. Londen, K. A. Meyer, and D. H. Sharpe (1980). Further experimentation on Taylor instability in solids. J. Appl. Phys., 51 . 4678-4679.

Cagliostro, D. J., D. A. Mandell, L. A. Schwalbe, T. F. Adams, and E. J. Chapyak (1990). MESA-3D calculations of armor penetration by projectiles with combined obliquity and yaw. Int. J. Impact Eng., 10, 81-92. 
Hemsing, W. F., A. R. Mathews, R. H. Warnes, and G. R. Whittenore (1991). VISAR: Line-imaging velocity interferometer. Seventh Topical Conference on Shock Compression in Condensed Matter, Williamsburg, VA.

Holian, K. S. (ed) (1984). T-4 handbook of material properties data base. Vol. IC: equations of state. Los Alamos National Laboratory report LA-10160-MS.

Johnson, G. R. and W. H. Cook (1985). Fracture characteristics of three metals subjected to various strains, strain rates, temperatures, and pressures. Eng. Fract ure Mech., 21, 31-48.

Johnson, G. R. and T. J. Holmquist (1989). Test data and computational strength and fracture model constants for 23 materials subjected to large strains, high strain ratus, and high temperatures. Los Alamos National Laboratory report LA-11463-MS.

Johnson, J. N. (1981). Dynamic fracture and spallation in ductile solids. J. Appl. Phys., 52, 2812-2825.

Kerrisk, J. F. and J. K. Meier (1991). Comparisons between Fast Shock Tube calculations and tests. Seventh Topical Conference on Shock Compressio: in Condensed Matter, Williamsburg, VA.

Marsh, S. P. and T. H. Tan (1991). Hypervelocity plate acceleration. Seventh Topical Conference on Shock Compression in Condensed Matter, Williamsburg, VA.

Me-Bar, Y., M. Boas, and Z. Rosenberg (1987). Spall studies on Ti-6Al-4V. Materials Sci. and Eng., 85, 77-84. 
Meier, J. K. and J. F. Kerrisk (1991). An introduction to the Fast Shock Tube (FST). Seventh Topisal Conference on Shock Compression in Condensed Matter, Williaınsburg, VA.

Menikoff, R., K. S. Lackner, N. L. Johnson, S. A. Colgate, J. M. Hyman, and G. A. Miranda (1991). Shock wave civen by a phased implosion. Phys. Fluids, $A 3,201-218$.

Swegle, J. W. and A. C. Robinson (1989). Acceleration instability in elastic-plastic solids. J. Appl. Phys. 66, 2838-2858. 


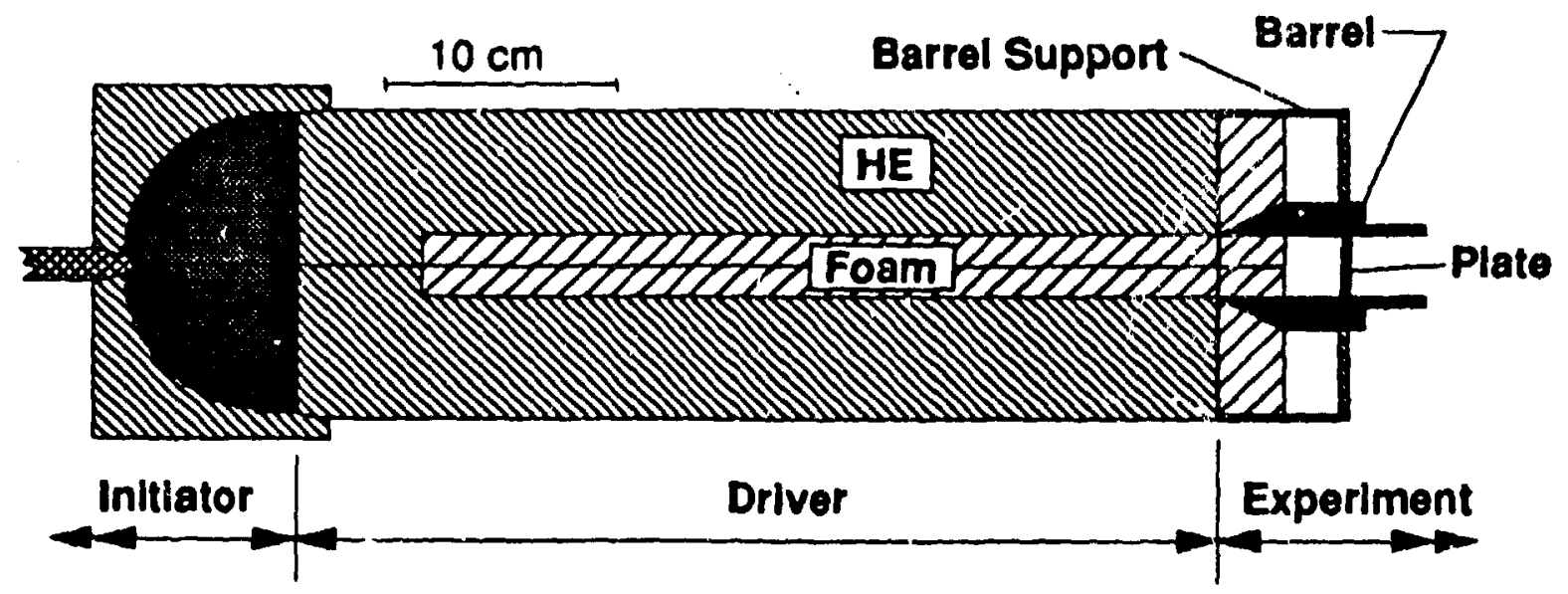

Fig. 1 Sketch of the Fast Shock Tube. 
PRESSURE (mbar)

time 45.0059

pr2/rung/Tinfst/sstiner/0.53fm7592/2mmssplate/2cmso/airtp/void/h1434/0.3mm cell

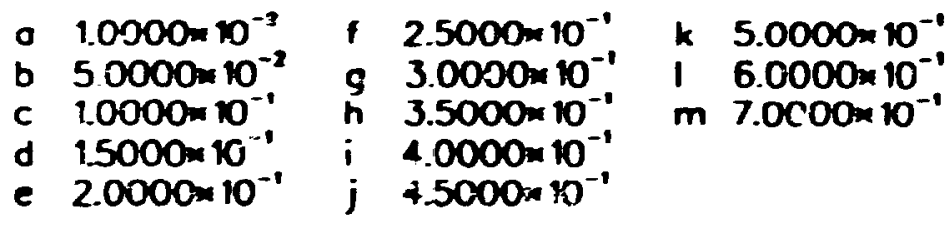

minimum $-1.16 \times 10^{-6}$

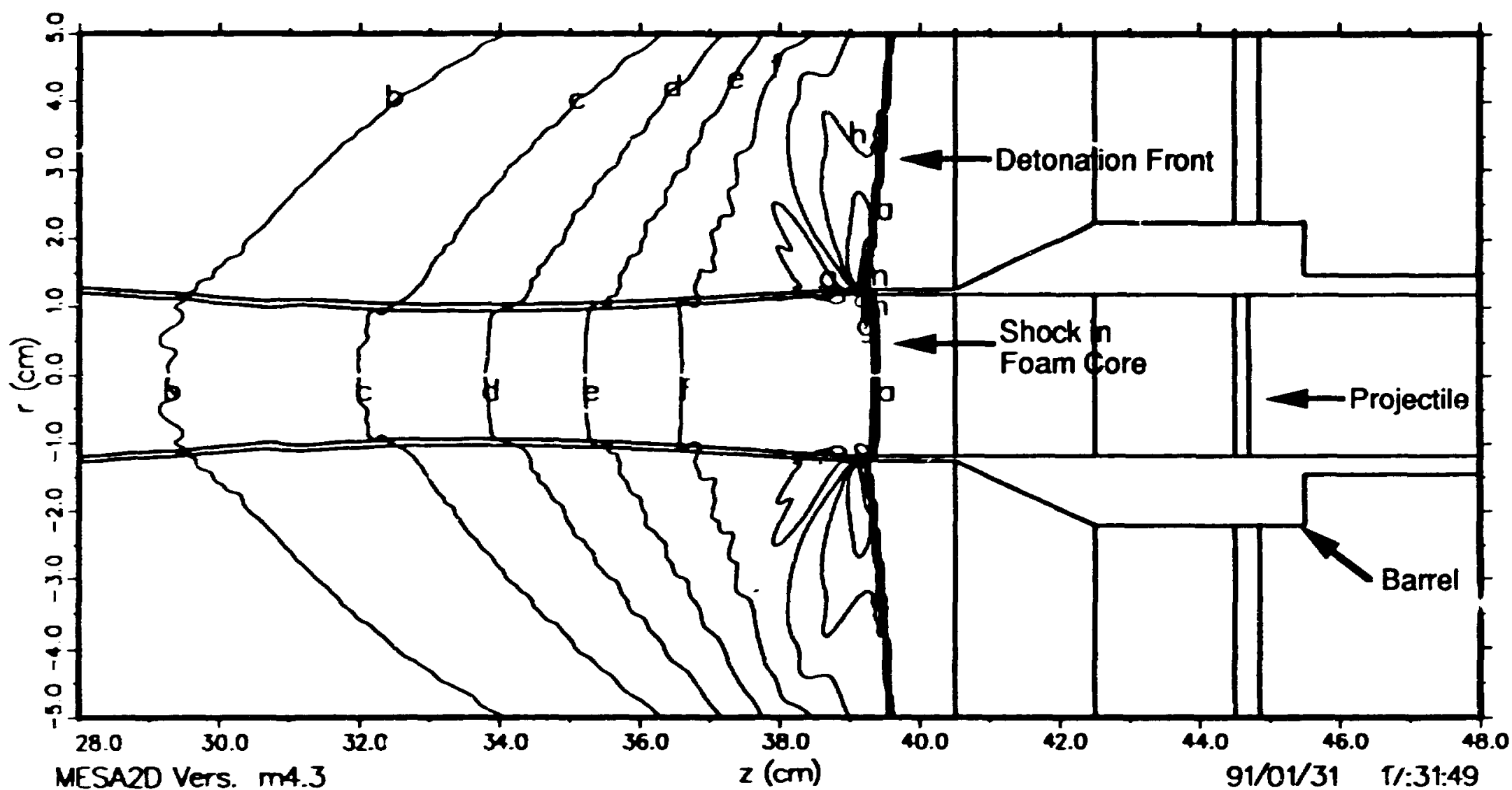

Fig. 2 Material interfaces and pressure contours in the FST at $45 \mu$ s after initiation of the driver HE. 
VECTOR VELOCTIES (cm/micro-sec)

time 55.0035

pr2/rung/infst/ssiner/0.53fm7592/2mmssplote/2cmso/oirto/void/41434/0.3mm cell

$\begin{array}{lrrr}\text { local max } & 8.1949 \times 10^{-1} & \text { globol max } & 8.1949 \times 10^{-1} \\ \longrightarrow & \text { scaled vec } & 8.1949 \times 10^{-1}\end{array}$

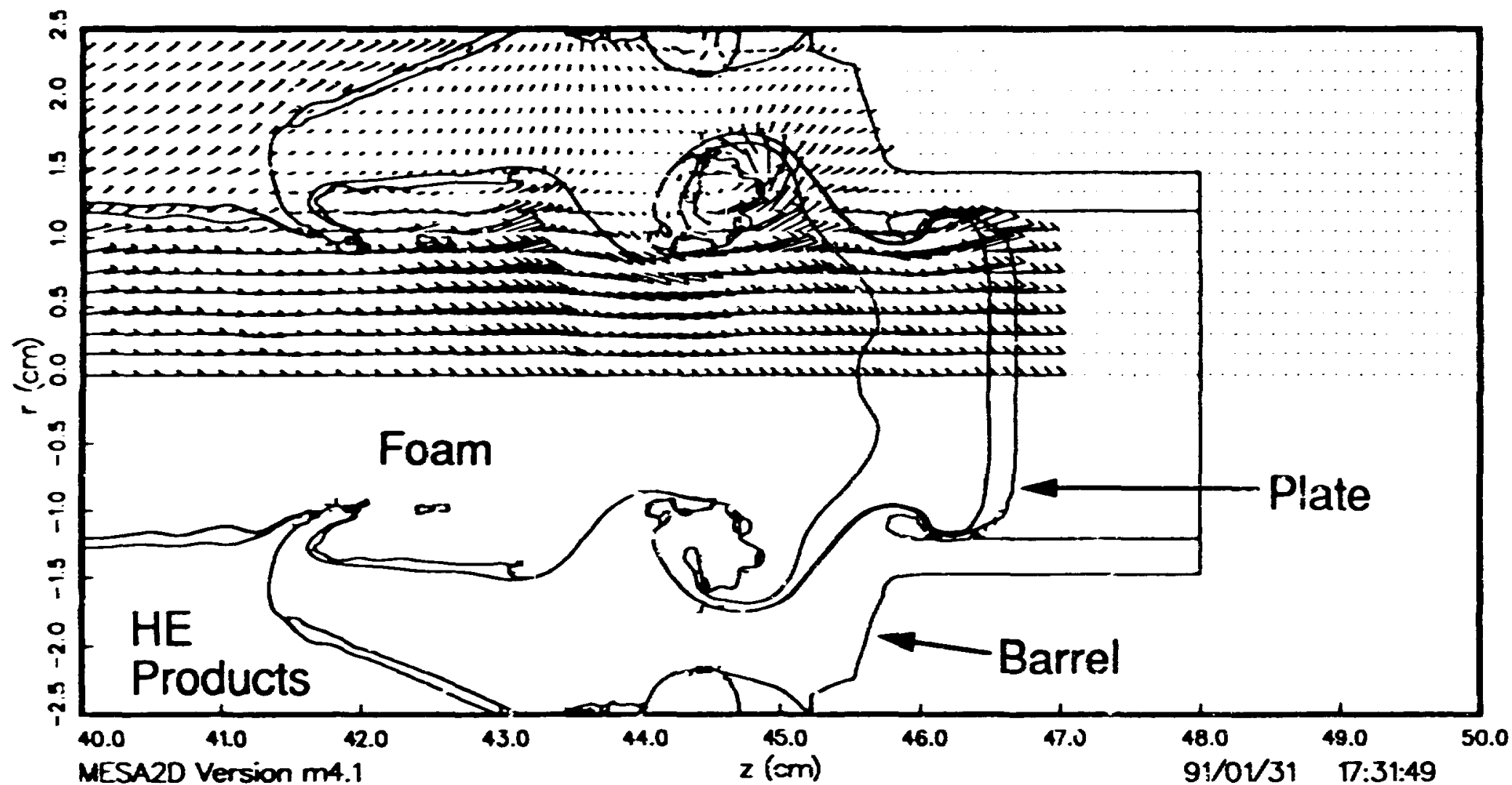

Fig. 3 Material interfaces and velocity vectors in the FST $\sim 5 \mu$ s after start of motion of the projectile. 

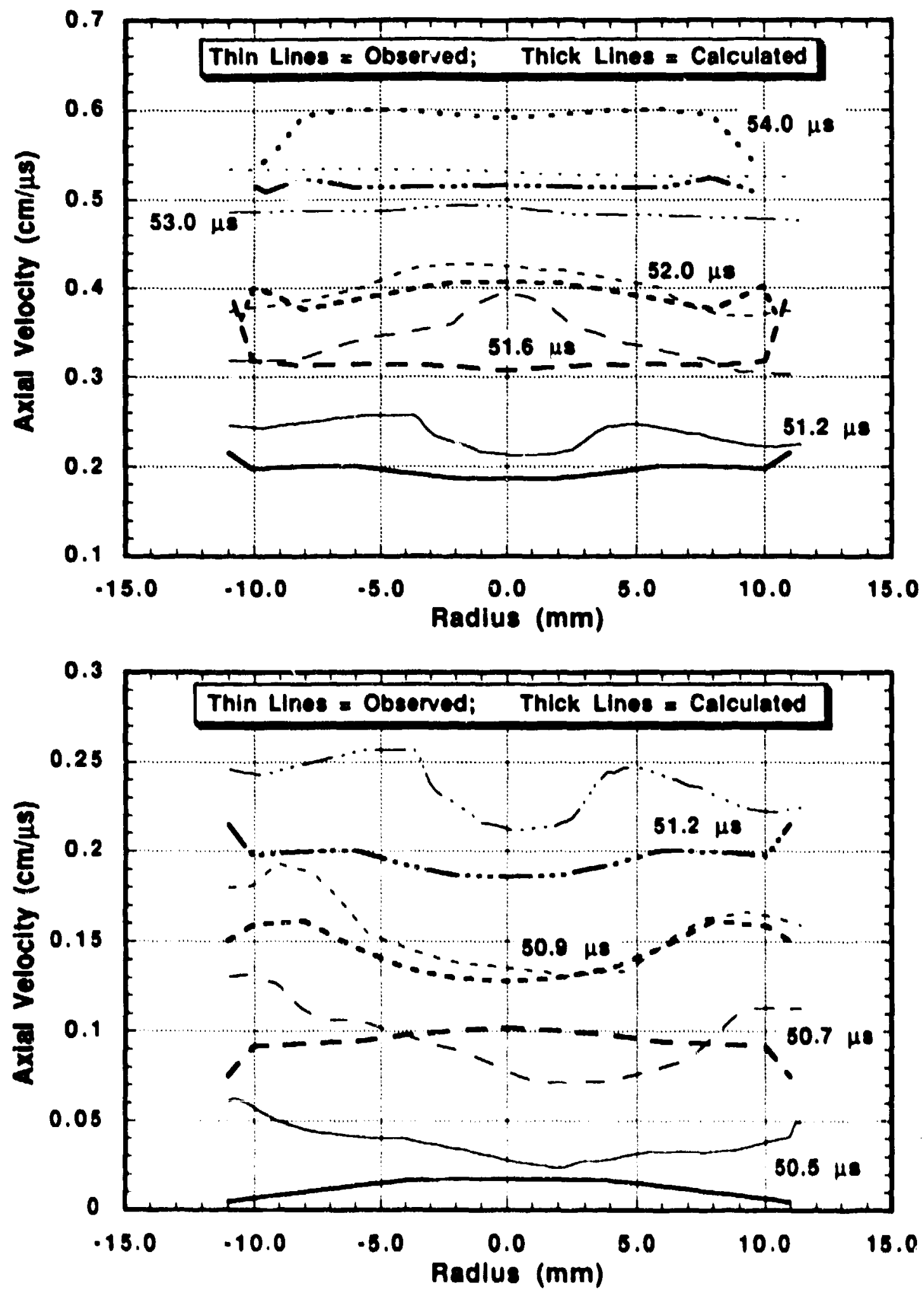

Fig. 4 Observed (LINE VISAR) and calculated (MESA/2D) velocity profiles of the front surface of a 2-mm thick stainless steel plate accelerated in the FST (test H1434). The lower plot shows times from 50.5 to $51.2 \mu \mathrm{s}$ on an expanded scale; the upper plot shows times from 51,2 to $54 \mu \mathrm{s}$. 


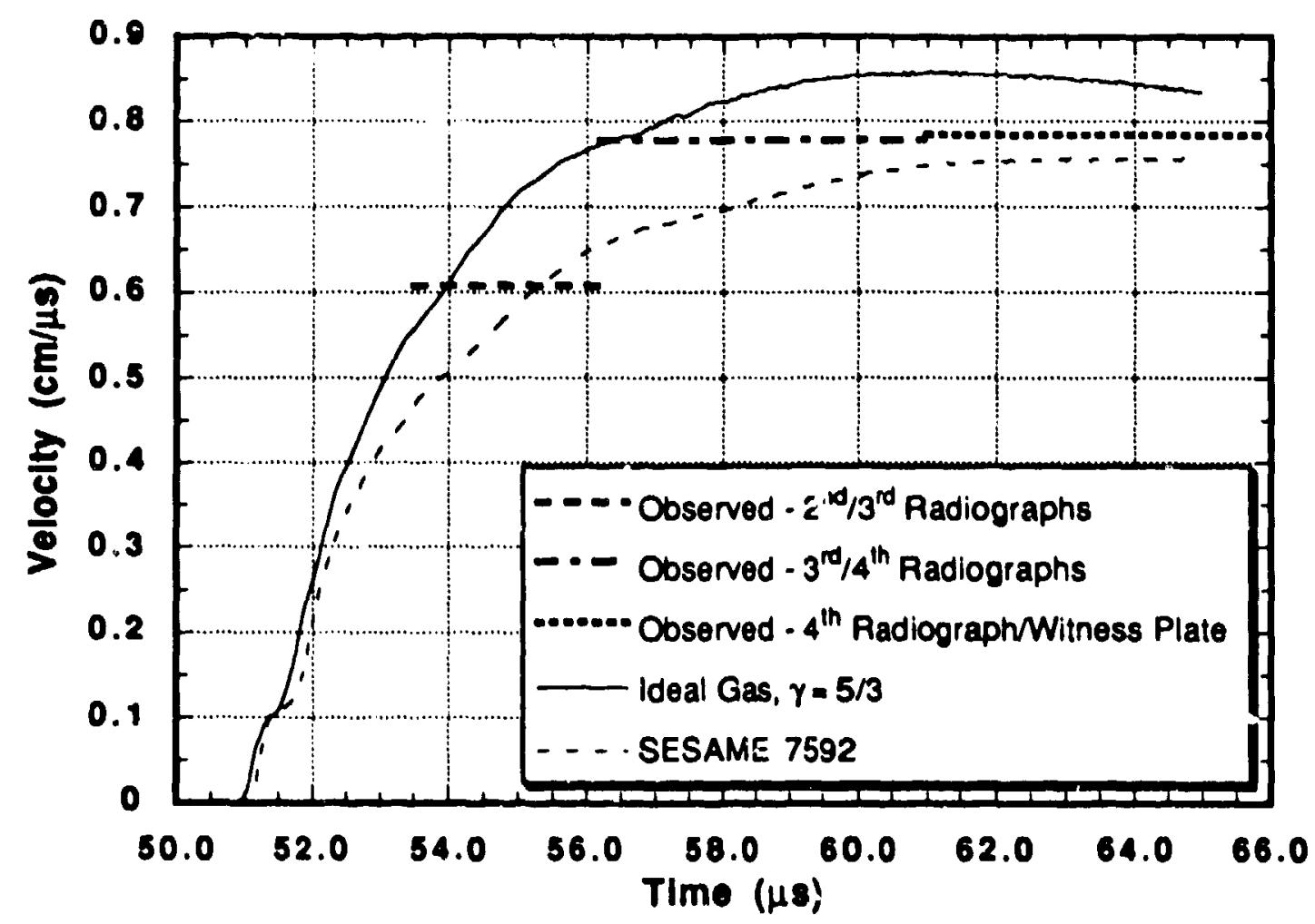

Fig. 5 Observed average projectile velocities obtained from radiographs and a witness plate compared with calculated (MESA2D) velocities using two foam EOSs (test E6035). 


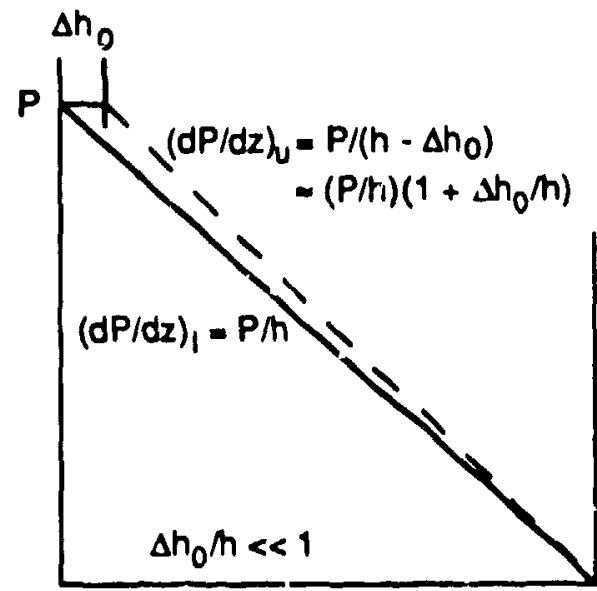

h

$$
\begin{aligned}
\frac{(d P / d z)_{u}}{(d P / d z)_{1}} & =\frac{(P / h)\left(1+\Delta h_{0} / h\right)}{(P / h)} \\
& =1+\Delta h_{0} m
\end{aligned}
$$

Varlable Surtace - Uniform Pressure

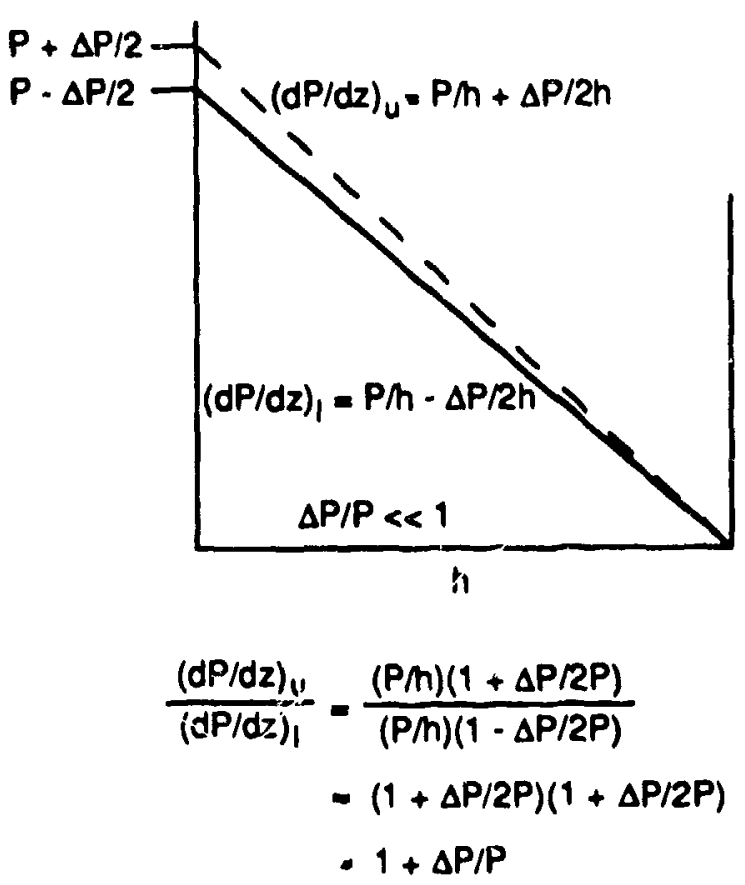

Uniform Surfacs - Variable Pressure

Fig. 6 Sketch of pressure gradients formed by a uniform pressure on a variable surface (left) and a variable pressure gradient on a uniform surface (right). 


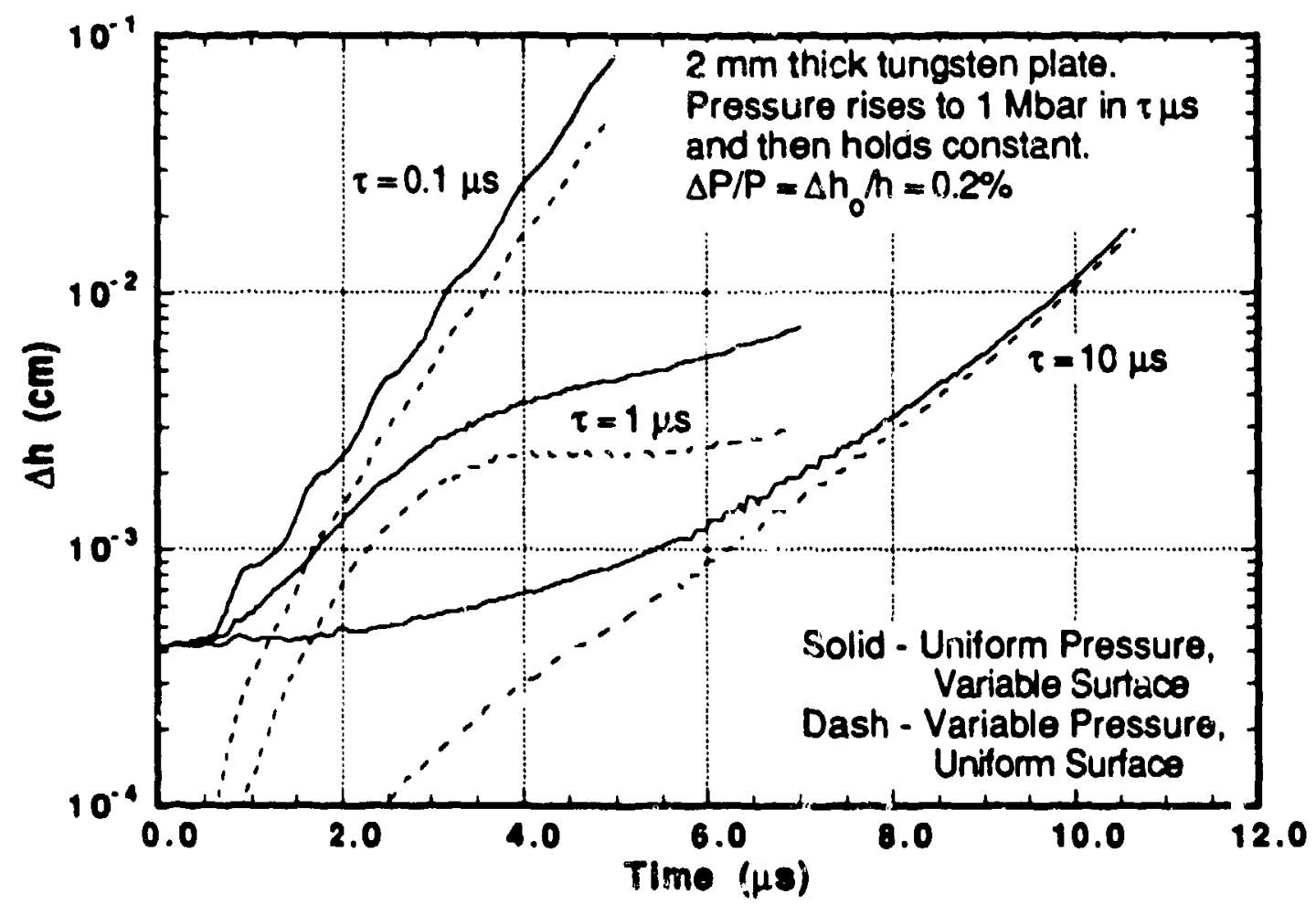

Fig. $7 \Delta$ h as a function of time for six calculations that compare the effects of initial surface perturbations with those of pressure perturbations on an Initially uniform surtace. 


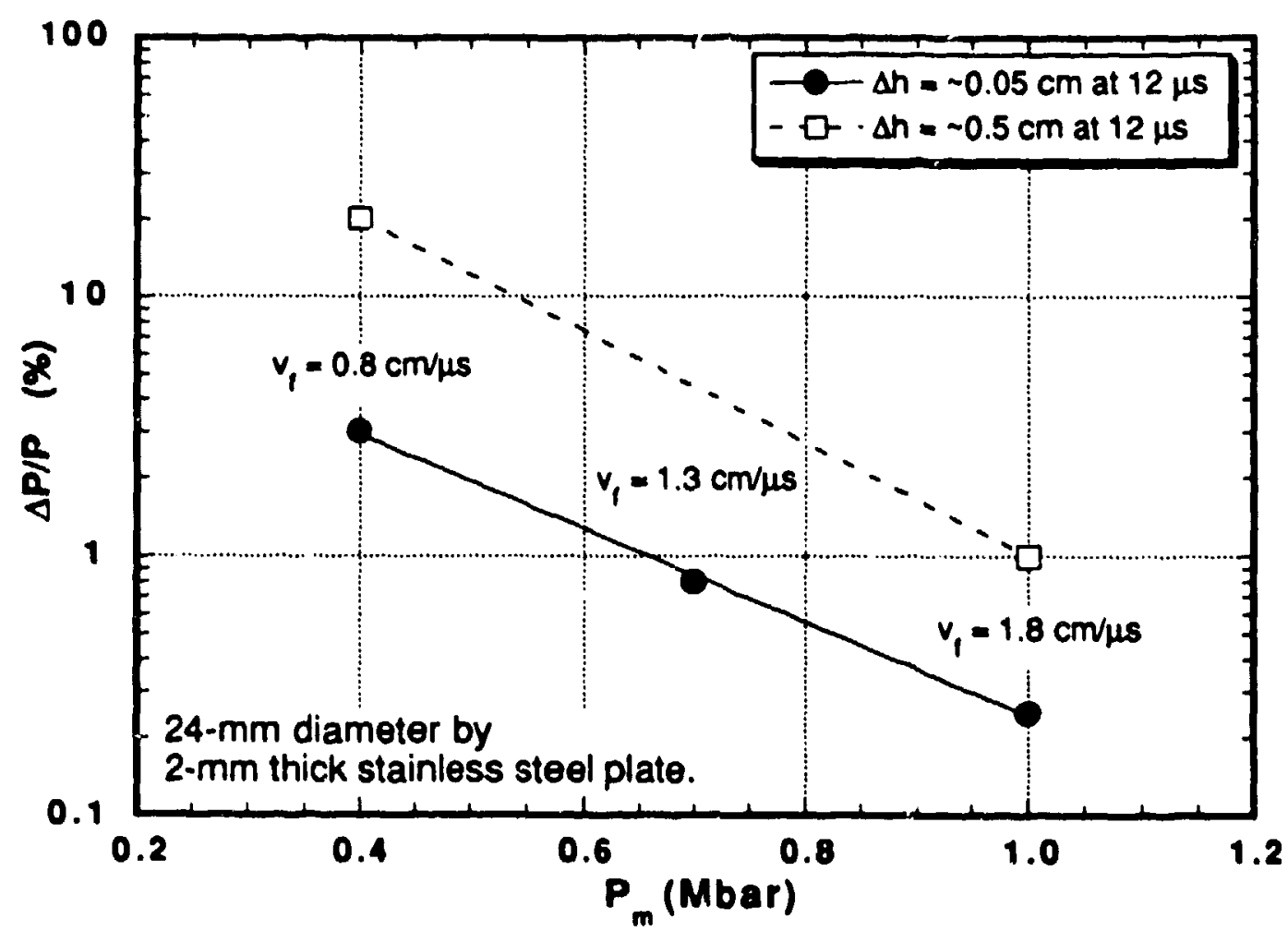

Fig. 8 Contour lines of constant $\Delta h_{m}$ at $12 \mu s$ as a function of $\Delta P / P$ and $P_{m}$ for the acceleration of a 2-mm thick stainless steel plate in the FST. The velocities are final plate velocities at peak driving pressures of $0.4,0.7$, and $1.0 \mathrm{Mbar}$. 


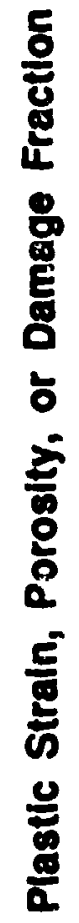

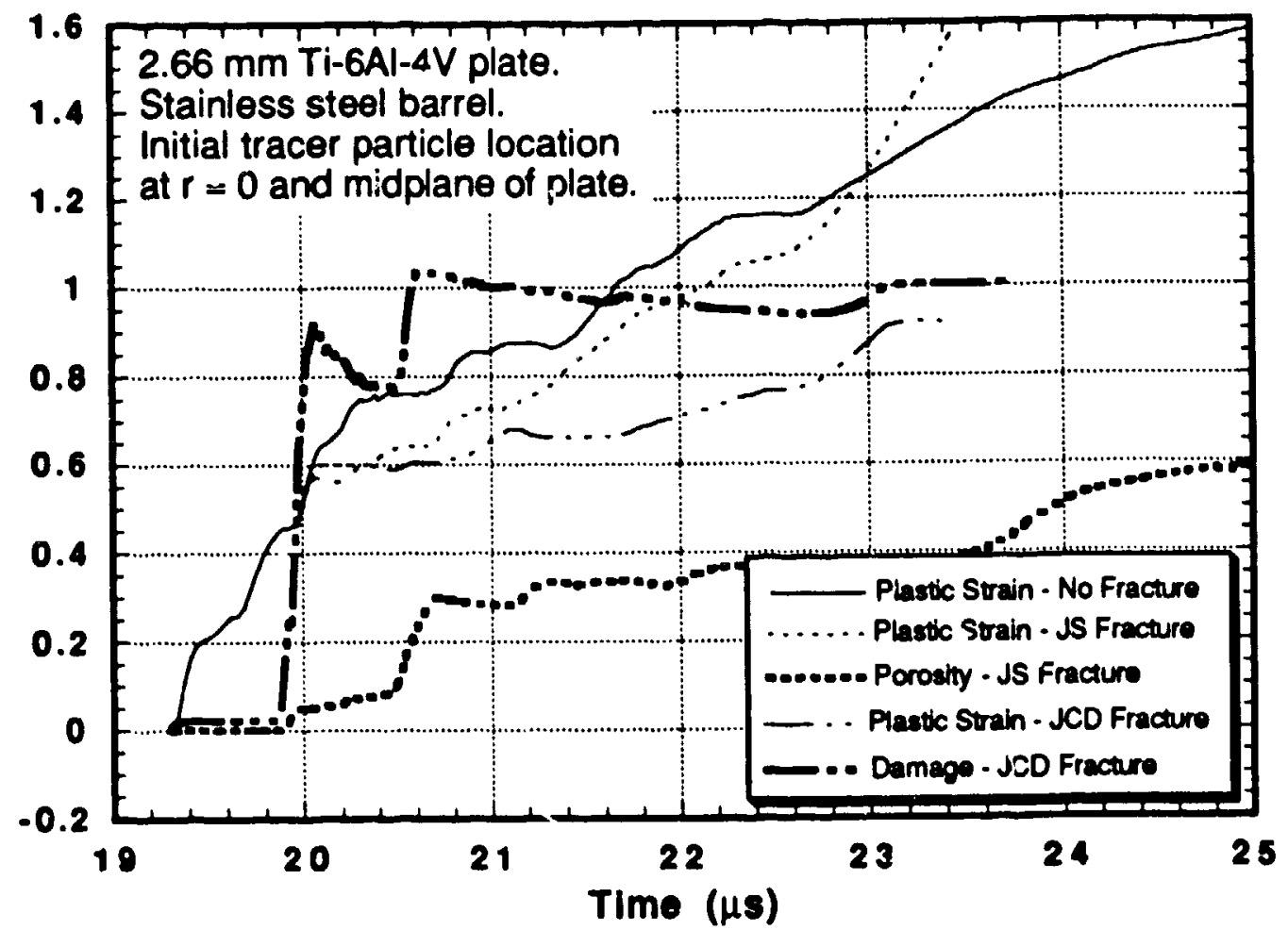

Fig. 9 Plastic strain, porosity (JS model), and damage fraction (JCD model) as a function of time for thrae calculations (without fracture, the JS model, and the JCD model.) of the acceleration of a $2.66-\mathrm{mm}$ thick Ti-6Al-4V plate. The three thin curves show plastic strains for the three calculations. The two thick curves show damage measures for the calculations using the JS and JCD models. 